யா

\title{
INTEGRATION OF THE SHORT-RANGE HYPERBOLIC SYSTEM JEMIOLUSZKA AND DGPS
}

\section{INTEGRACJA HIPERBOLICZNEGO SYSTEMU BLISKIEGO ZASIĘGU JEMIOLUSZKA Z DGPS}

\author{
Andrzej Banachowicz ${ }^{1}$, Adam Wolski ${ }^{2}$, Grzegorz Banachowicz ${ }^{3}$ \\ (1) Gdynia Maritime Academy, Faculty of Navigation \\ Akademia Morska w Gdyni, Katedra Nawigacji \\ Aleja Zjednoczenia 3, 81-345 Gdynia, Poland \\ $(2,3)$ Maritime University of Szczecin, Faculty of Navigation \\ Akademia Morska w Szczecinie, Instytut Nawigacji Morskiej \\ ul. Waly Chrobrego 1-2, 70-500 Szczecin, Poland
}

e-mails: (1) andrzejbanachowicz@wp.pl, (2) wolski@am.szczecin.pl,

(3) gbanachowicz@am.szczecin.pl

\begin{abstract}
This article describes an algorithm of an integrated navigational system developed for the navigation and steering along a given trajectory by rescuesalvage ships operated by the Polish Navy. Due to strict requirements concerning the accuracy of measurements of navigational parameters of ships at slow speeds, inertial measurement units have been used, whilst the state variables in the algorithm include higher derivatives of the velocity vector. The algorithm has been verified by a simulation method and at sea trials on board a real ship.
\end{abstract}

Keywords: DGPS, safety of navigation.

Streszczenie. W artykule przedstawiono opis algorytmu zintegrowanego systemu nawigacyjnego opracowanego dla potrzeb nawigacji i sterowania po trajektorii specjalistycznych okrętów wojennych. Ze względu na wysokie potrzeby dokładnościowe mierzonych parametrów nawigacyjnych na małych prędkościach, wykorzystano $\mathrm{w}$ systemie mierniki inercjalne, a $\mathrm{w}$ algorytmie jako zmienne stanu przyjęto również wyższe pochodne wektora prędkości. Algorytm zweryfikowano metodą symulacyjną oraz podczas prób morskich na obiekcie rzeczywistym.

Słowa kluczowe: DGPS, bezpieczeństwo nawigacji. 


\section{INTEGRATION OF THE SHORT-RANGE HYPERBOLIC SYSTEM JEMIOLUSZKA AND DGPS}

\section{Introduction}

Certain initial assumptions are made while developing algorithms of an integrated navigational system (INS). Some of these assumptions are as follows:

- the system should work in various configurations of the equipment (in order to ensure reliability), from the simplest dead reckoning (DR) with the use of a calculator (data put in manually), through an autonomous automated dead reckoning, to a fully integrated system combining all sources of navigational measurements available;

- the uniform reference system should be adopted, such as WGS-84 (World Geodetic System), where position coordinates can be transformed to a local datum;

- measurements should be tested (quality control) in order to eliminate significant errors referred to as outliers;

- measurements of specified stationary equipment and navigational systems are used for flexible algorithms, with defined navigational parameters and their transmission protocols;

- measurement acquisition may be synchronic or asynchronic;

- methods of measurement estimation and integration should be defined;

- the final user should specify other requirements, if any.

Navigation and automatic steering of special warships along a given trajectory requires high accuracy and integrity of navigational information. This issue becomes particularly significant when these requirements have to be satisfied within the entire range of a ship's manoeuvring speed, i.e. from zero to maximum speed. Among others, ships that actually operate at very slow or maximum speeds are, minehunters and rescue-salvage ships.

This article describes elements of an integrated navigational system implemented in rescue-salvage ships of the Polish Navy. As these ships require high accuracy of navigational position determination, even when the ship moves at slow speed close to zero in the dynamic positioning $(D P)$, the 
following configuration of navigational systems and equipment has been adopted [1]:

- gyrocompass,

- inertial measurement unit,

- Doppler log,

- (D)GPS receiver,

- short-range shore based radionavigational system receiver (JEMIOLUSZKA).

The algorithm of navigational parameters integration is based on an Extended Kalman Filter - EKF, where ship's movement parameters (its dynamics) are estimated.

\section{JEMIOLUSZKA}

JEMIOLUSZKA is a land-based hyperbolic radionavigation system. Pilot and hydrographic services have used the JEMIOLUSZKA system for many years in the Gdansk Bay [3]. The JEMIOLUSZKA system works at $1800 \mathrm{kHz}$ frequency band. The range of this system is 150 nautical miles (on surface wave) and the average error of position is not greater than $7 \mathrm{~m}$. The JEMIOLUSZKA system can work in different variants:

- as mobile or stationary system,

- as one master station and two or more slave stations,

- as an independent pair of stations.

Figures 1 and 2 show examples of station configuration. In both figures the system is installed in Gdansk Bay. In Figure 1 the chain consists of a master station installed in Gdansk and slave stations installed in Jastarnia and Krynica Morska.

Figure 2 shows a chain consisting of two pairs: Gdynia - Hel and Kuznica - Gorki Zachodnie. This installation provides an optimal configuration minimization of the HDOP (Horizontal Dilution of Precision) for most important parts of Gdansk Bay in terms of navigational safety. 


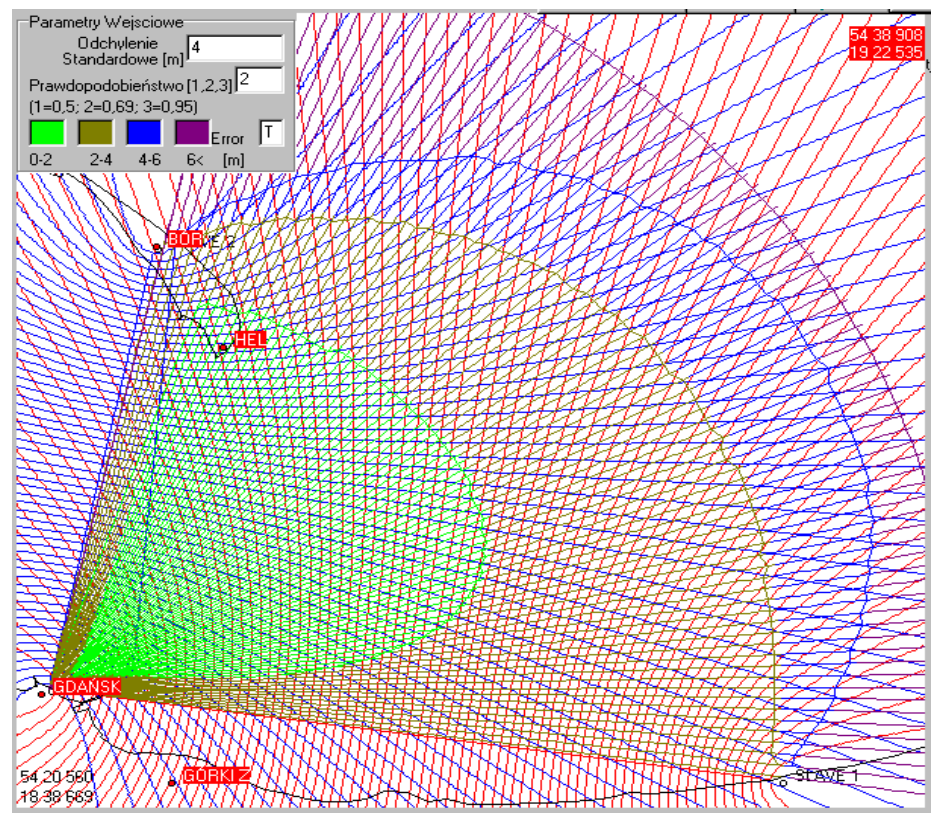

Fig. 1. Configuration - one master station and two slave stations

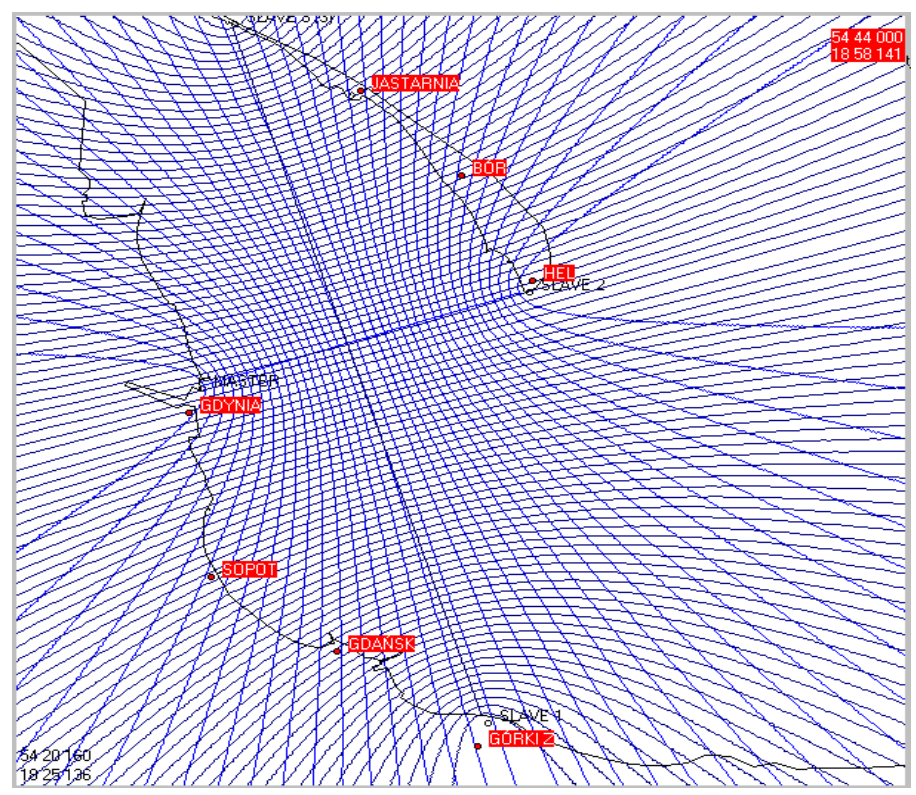

Fig. 2. Configuration - two independent pairs of stations 


\section{Filter parameters}

As assumed above, the filter will use measurements from the following navigational systems and instruments [1]:

- satellite navigational system DGPS - position coordinates $\left(\varphi_{D G P S}, \lambda_{D G P S}\right)$,

- short-range hyperbolic radionavigational system JEMIOLUSZKA position coordinates $\left(\varphi_{J E M}, \lambda_{J E M}\right)$,

- Doppler log-velocity components relative to the bottom $\left(V_{x}, V_{y}\right)$,

- gyrocompass - ship's course $(C)$,

- inertial measurement unit - components of acceleration relative to the bottom $\left(a_{x}, a_{y}\right)$.

The measured components of velocity and acceleration are converted projections of velocity vector relative to the bottom on the meridian and parallel $\left(V_{N}, V_{E}\right)$, projections of the acceleration vector relative to the bottom on the meridian and parallel $\left(a_{N}, a_{E}\right)$ and projections of jerks relative to the bottom on the meridian and parallel $\left(j_{N}, j_{E}\right)$. A jerk is considered as white noise.

In this case the state vector will have the following elements:

$$
\mathbf{x}=\left[\varphi, \lambda, V_{N}, V_{E}, a_{N}, a_{E}, j_{N}, j_{E}\right]^{\mathrm{T}}
$$

The transition matrix will be defined as follows:

$$
\mathbf{A}_{i+1, i}=\left[\begin{array}{cccccccc}
1 & 0 & \Delta t_{i} & 0 & \Delta t_{i}{ }^{2} / 2 & 0 & \Delta t_{i}^{3} / 6 & 0 \\
0 & 1 & 0 & \Delta t_{i} & 0 & \Delta t_{i}^{2} / 2 & 0 & \Delta t_{i}^{3} / 6 \\
0 & 0 & 1 & 0 & \Delta t_{i} & 0 & \Delta t_{i}{ }^{2} / 2 & 0 \\
0 & 0 & 0 & 1 & 0 & \Delta t_{i} & 0 & \Delta t_{i}^{2} / 2 \\
0 & 0 & 0 & 0 & 1 & 0 & \Delta t_{i} & 0 \\
0 & 0 & 0 & 0 & 0 & 1 & 0 & \Delta t_{i} \\
0 & 0 & 0 & 0 & 0 & 0 & 1 & 0 \\
0 & 0 & 0 & 0 & 0 & 0 & 0 & 1
\end{array}\right]
$$

where: $\Delta t_{i}=t_{i+1}-t_{i}$. 
Now the evolution of the state is determined by derivatives of higher orders of particular navigational parameters. The covariance matrix of state disturbances has a form adjusted to the elements of vector state. Therefore we obtain:

$$
\mathbf{Q}_{i}=\left[\begin{array}{cccccccc}
\sigma_{\varphi}^{2}+\Delta t_{i}{ }^{2} \sigma_{V_{\varphi}}^{2} & \Delta t_{i}{ }^{2} \sigma_{V_{\varphi} V_{\lambda}} & 0 & 0 & 0 & 0 & 0 & 0 \\
\Delta t_{i}{ }^{2} \sigma_{V_{\varphi} V_{\lambda}} & \sigma_{\lambda}^{2}+\Delta t_{i}^{2} \sigma_{V_{\lambda}}^{2} & 0 & 0 & 0 & 0 & 0 & 0 \\
0 & 0 & \sigma_{V_{N}}^{2} & \sigma_{V_{N} V_{E}} & 0 & 0 & 0 & 0 \\
0 & 0 & \sigma_{V_{N} V_{E}} & \sigma_{V_{N}}^{2} & 0 & 0 & 0 & 0 \\
0 & 0 & 0 & 0 & \sigma_{a_{N}}^{2} & \sigma_{a_{N} a_{E}} & 0 & 0 \\
0 & 0 & 0 & 0 & \sigma_{a_{N} a_{E}} & \sigma_{a_{E}}^{2} & 0 & 0 \\
0 & 0 & 0 & 0 & 0 & 0 & \sigma_{j_{N}}^{2} & 0 \\
0 & 0 & 0 & 0 & 0 & 0 & 0 & \sigma_{j_{E}}^{2}
\end{array}\right]
$$

Measured quantities in this model are the coordinates of system position DGPS $\left(\varphi_{D G P S}, \lambda_{D G P S}\right)$, system position coordinates of JEMIOLUSZKA $\left(\varphi_{J E M}, \lambda_{J E M}\right)$, components of velocity relative to the meridian and parallel obtained by dead reckoning $\left(V_{N}, V_{E}\right)$ and components of acceleration relative to the meridian and parallel obtained from the inertial measurement unit $\left(a_{N}, a_{E}\right)$. With these assumptions, the measurement vector will have this form:

$\mathbf{z}=\left[\varphi_{D G P S}, \lambda_{D G P S}, \varphi_{J E M}, \lambda_{J E M}, V_{N}, V_{E}, a_{N}, a_{E}\right]^{\mathrm{T}}$

The measurement matrix is the Jacobi matrix

$$
\mathbf{C}=\left[\begin{array}{cccccccc}
1 & 0 & 0 & 0 & 0 & 0 & 0 & 0 \\
0 & 1 & 0 & 0 & 0 & 0 & 0 & 0 \\
\frac{\partial \varphi_{J E M}}{\partial \varphi} & 0 & 0 & 0 & 0 & 0 & 0 & 0 \\
0 & \frac{\partial \lambda_{J E M}}{\partial \lambda} & 0 & 0 & 0 & 0 & 0 & 0 \\
0 & 0 & 1 & 0 & 0 & 0 & 0 & 0 \\
0 & 0 & 0 & 1 & 0 & 0 & 0 & 0 \\
0 & 0 & 0 & 0 & 1 & 0 & 0 & 0 \\
0 & 0 & 0 & 0 & 0 & 1 & 0 & 0
\end{array}\right]
$$


The covariance matrix of measurement disturbances (measurement vector) in this case will be as follows:

$$
\mathbf{R}=\left[\begin{array}{cccccccc}
\sigma_{\varphi_{D G P S}}^{2} & \sigma_{\varphi \lambda_{D G P S}} & 0 & 0 & 0 & 0 & 0 & 0 \\
\sigma_{\varphi \lambda_{D G P S}} & \sigma_{\lambda_{D G P S}}^{2} & 0 & 0 & 0 & 0 & 0 & 0 \\
0 & 0 & \sigma_{\varphi_{J E M}}^{2} & \sigma_{\varphi \lambda_{J E M}} & 0 & 0 & 0 & 0 \\
0 & 0 & \sigma_{\varphi \lambda_{J E M}} & \sigma_{\lambda_{J E M}}^{2} & 0 & 0 & 0 & 0 \\
0 & 0 & 0 & 0 & \sigma_{V_{N}}^{2} & \sigma_{V_{N} V_{E}} & 0 & 0 \\
0 & 0 & 0 & 0 & \sigma_{V_{N} V_{E}} & \sigma_{V_{E}}^{2} & 0 & 0 \\
0 & 0 & 0 & 0 & 0 & 0 & \sigma_{a_{N}}^{2} & \sigma_{a_{N} a_{E}} \\
0 & 0 & 0 & 0 & 0 & 0 & \sigma_{a_{N} a_{E}} & \sigma_{a_{E}}^{2}
\end{array}\right]
$$

\section{Navigational parameter measurement accuracy}

The covariance matrix consists of variances and covariances of particular navigational measurements. These characteristics were determined during static (laboratory) tests and at sea. Figure 1 shows an example of changes in geographic coordinates obtained from a DGPS.

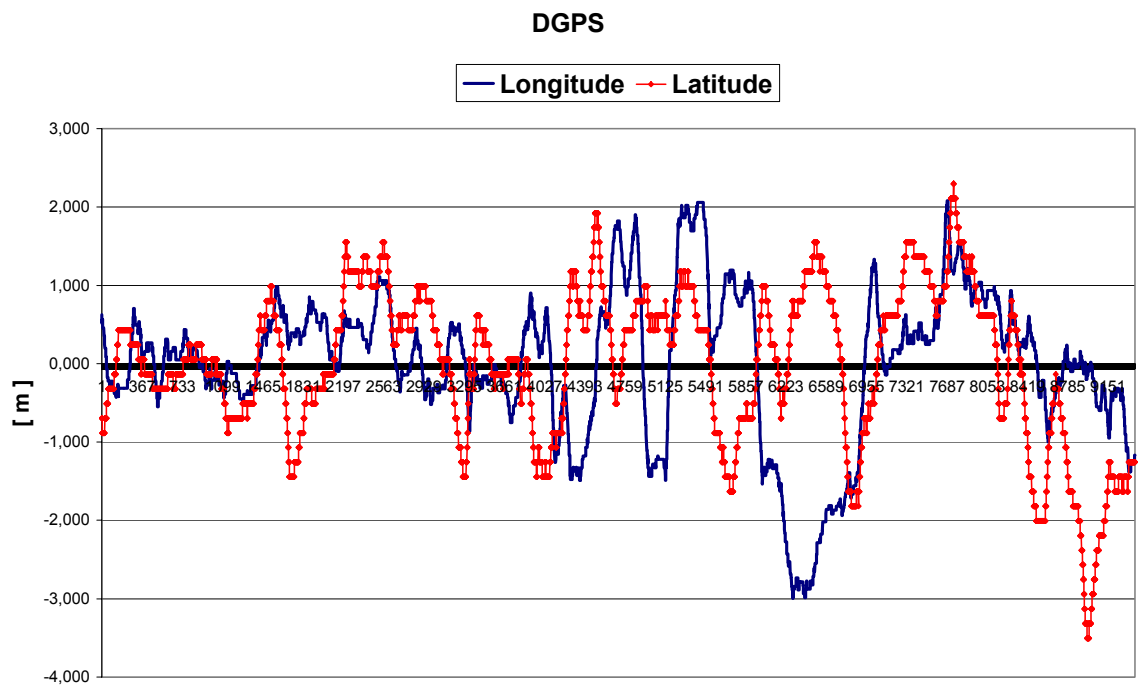

Fig. 3. Changes in geographic coordinates of a DGPS 
The same kind of data referring to the JEMIOLUSZKA system are presented in Figure 4.

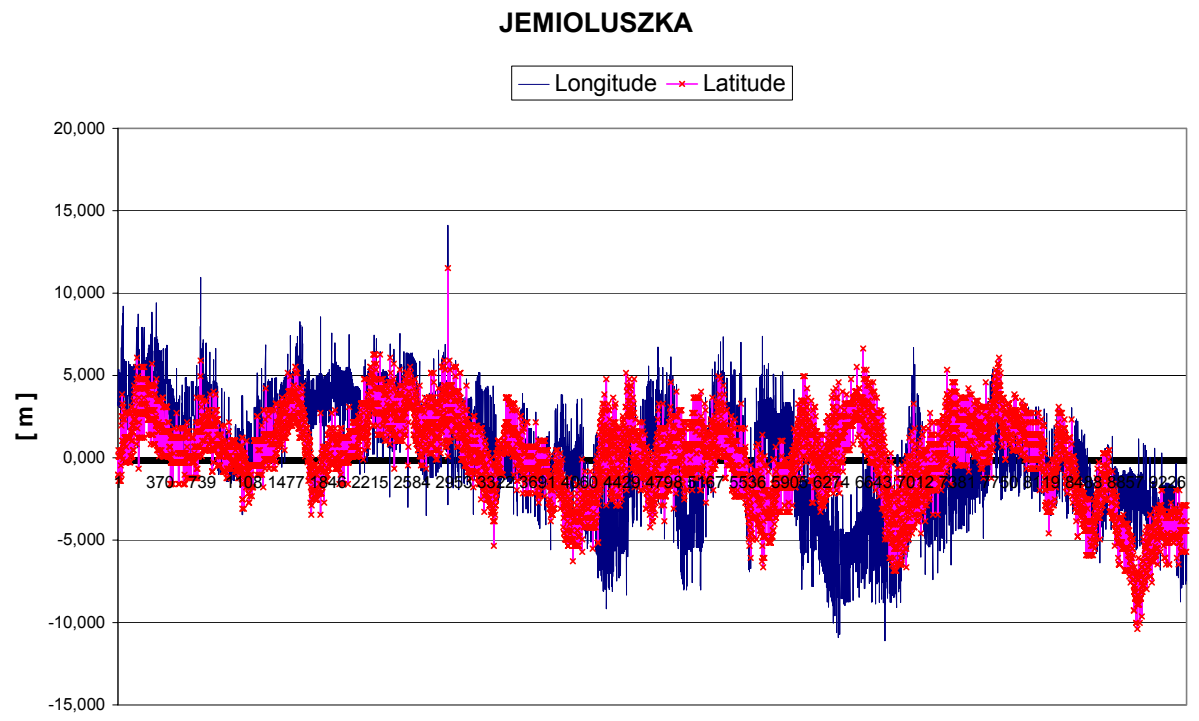

Fig. 4. Changes in geographic coordinates of the JEMIOLUSZKA system

As a result, the following values of variance and covariance of the measurements were adopted:

- DGPS system $-\sigma_{\varphi_{D G P S}}^{2}=4.0\left[\mathrm{~m}^{2}\right], \sigma_{\lambda_{D G P S}}^{2}=2,25\left[\mathrm{~m}^{2}\right]$, the coordinates are not correlated;

- velocity components $-\sigma_{V}=0.1 \mathrm{~m} / \mathrm{s}$;

- acceleration components $-\sigma_{a}=0.01 \mathrm{~m} / \mathrm{s}^{2}$;

- JEMIOLUSZKA system [2], [3]:

a) variance of geographic latitude determination

$$
\sigma_{\varphi_{J E M}}^{2}=\frac{1}{4} \sigma_{\Delta D}^{2} \csc ^{2} \theta\left(\cos ^{2} A_{12} \csc ^{2} \frac{\omega_{23}}{2}+\cos ^{2} A_{23} \csc ^{2} \frac{\omega_{12}}{2}\right)
$$

b) variance of geographic longitude determination 


$$
\sigma_{\lambda_{J E M}}^{2}=\frac{1}{4} \sigma_{\Delta D}^{2} \csc ^{2} \theta\left(\sin ^{2} A_{12} \csc ^{2} \frac{\omega_{23}}{2}+\sin ^{2} A_{23} \csc ^{2} \frac{\omega_{12}}{2}\right),
$$

c) covariance between geographic coordinates:

$$
\sigma_{\varphi \lambda_{J E M}}=\frac{1}{8} \sigma_{\Delta D}^{2} \csc ^{2} \theta\left(\sin \left(A_{1}+A_{2}\right) \csc ^{2} \frac{\omega_{23}}{2}+\sin \left(A_{2}+A_{3}\right) \csc ^{2} \frac{\omega_{12}}{2}\right) ;
$$

where:

$A_{i j}-$ average azimuth between the $i$-th and the $j$-th station,

$\omega_{i j}$ - base angle between the $i$-th and the $j$-th station,

$\sigma_{\Delta \mathrm{D}}-$ measurement error of distance difference,

$$
\theta=A_{i j}-A_{j k}
$$

\section{Conclusion}

The described algorithm of navigational parameters estimation has been examined in a simulation research, then verified at sea trials. The latter have shown that the filter works correctly and is useful in an INS algorithm of specialized ships. Figure 5 shows a comparison of positions obtained from an integrated navigational system (INS) with those received from positioning systems: DGPS and JEMIOLUSZKA. 


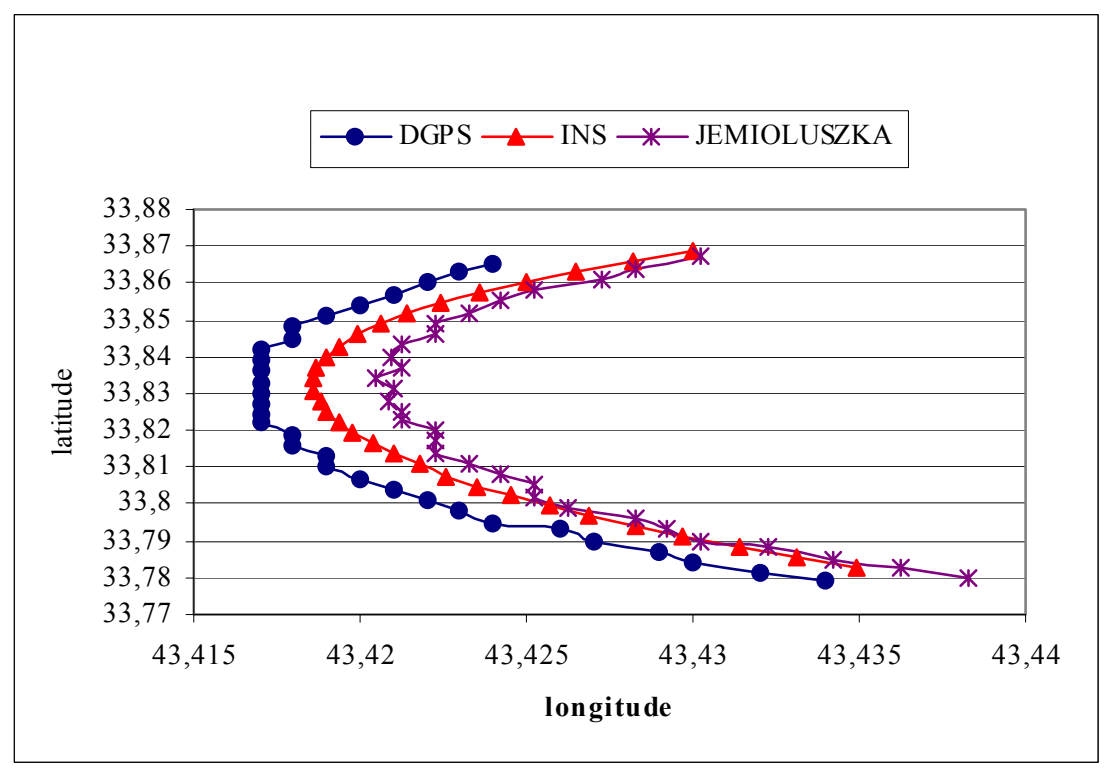

Fig. 5. A comparison of positions obtained by DGPS, JEMIOLUSZKA and INS - ship's turning manoeuvre

The presented model of the navigational process is just one of possible solutions. It should be emphasized that the form of a particular model to a large extent contributes to a success or failure of a new filter developed. This especially concerns a correct and realistic determination of elements of the particular matrices - transition $\mathbf{A}$, measurements $\mathbf{C}$ and covariance matrices $\mathbf{Q}$ and $\mathbf{R}$. In the case of the covariance matrix the ratio of adequate elements seems to be the most important. If too large errors of disturbances are adopted, the filter becomes 'stiff' and excessively follows the measurements [1], [4]. The effect is that measurement errors are not filtered. If, on the other hand, too small values of state disturbance are adopted, the filter will reject measurements differing too much from forecast values. This is particularly important in real measurements when there are differences between measurements from various navigational instruments and systems and when both measuring instruments and measurement results are not reliable. Besides, the problems of time scale synchronization, length of measuring cycles and discretization intervals have to be taken into consideration. 


\section{References}

1. Banachowicz A., Bober R.: Integrated navigation system for the Polish warships Piast and Lech. (in Polish). Maritime University of Szczecin, Szczecin, 1999.

2. Banachowicz A.: Variants of Structural and Measurement Models of an Integrated Navigational System. Annual of Navigation No 3, Gdynia, 2001.

3. Banachowicz A., Kamiński R.: Analysis Accuracy Integrated Position of Hyperbolic System JEMIOLUSZKA and Satellite Navigation System GPS. GEOINFORMATICS 2004. $12^{\text {th }}$ International Conference on Geoinformatics "Geospatial information Research: Bridging the Pacific and Atlantic". Sweden, Gävle 7-9 June 2004.

4. Farrell J.A., Barth M.: The Global Positioning System \& Inertial Navigation. McGraw-Hill, New York, 1999. 


\section{INTEGRACJA HIPERBOLICZNEGO SYSTEMU BLISKIEGO ZASIĘGU JEMIOLUSZKA Z DGPS}

\section{Wprowadzenie}

Opracowując algorytmy zintegrowanego systemu nawigacyjnego (ZSN) przyjmuje się pewne ogólne założenia wstępne. Oto niektóre z nich:

- system powinien pracować $\mathrm{w}$ różnych konfiguracjach sprzętowych (względy niezawodnościowe), poczynając od najprostszej - zliczenia drogi w wersji kalkulatorowej (dane wprowadzane z klawiatury), poprzez autonomiczną zautomatyzowaną nawigację zliczeniową a kończąc na układzie w pełni zintegrowanym łączącym wszystkie dostępne źródła pomiarów nawigacyjnych;

- należy przyjąć jednolity układ odniesienia - WGS-84 (World Geodetic System), z możliwością transformacji współrzędnych pozycji do układów lokalnych,

- pomiary powinny być testowane w celu eliminacji grubych błędów,

- wykorzystujemy pomiary stałych wyspecyfikowanych urządzeń i systemów nawigacyjnych lub algorytmy elastyczne z określeniem parametrów nawigacyjnych i protokołów ich transmisji,

- akwizycja pomiarów synchroniczna lub asynchroniczna,

- metody estymacji i integracji pomiarów,

- inne wymagania określone przez zamawiającego.

Nawigacja i automatyczne sterowanie po trajektorii specjalistycznymi okrętami wojennymi wymaga wysokiej dokładności i wiarygodności informacji nawigacyjnej. Szczególnego znaczenia nabiera ten problem $\mathrm{w}$ przypadku konieczności spełnienia tych wymagań w pełnym zakresie prędkości manewrowania okrętu, tj. od prędkości zerowej do maksymalnej. Taka sytuacja ma miejsce między innymi w nawigacji okrętów zwalczania min oraz okrętów ratowniczych.

W niniejszym artykule opisano elementy zintegrowanego systemu nawigacyjnego wdrożonego na okrętach ratowniczych Marynarki Wojennej RP. Ponieważ wymagana jest dla nich duża dokładność określania parametrów nawigacyjnych także dla prędkości bliskich zeru w reżimie 
dynamicznej stabilizacji pozycji, przyjęto następującą konfigurację urządzeń i systemów nawigacyjnych [3]:

- żyrokompas,

- przetwornik inercjalny (inertial measurement unit - IMU),

- $\log$ Dopplerowski,

- odbiornik (D)GPS,

- odbiornik naziemnego radionawigacyjnego systemu bliskiego zasięgu JEMIOLUSZKA.

Algorytm integracji parametrów nawigacyjnych opiera się na rozszerzonym filtrze Kalmana (Extended Kalman Filter - EKF), w którym estymowane są parametry ruchu statku (jego dynamika).

\section{JEMIOLUSZKA}

JEMIOŁUSZKA jest naziemnym hiperbolicznym systemem radionawigacyjnym. Piloci oraz służby hydrograficzne od wielu lat wykorzystują go na Zatoce Gdańskiej [3]. System JEMIOŁUSZKA pracuje w paśmie częstotliwości $1800 \mathrm{kHz}$. Zasięg systemu 150 mil morskich (na fali powierzchniowej), a błąd określenia pozycji jest nie większy od 7 metrów. JEMIOŁUSZKA może pracować w różnych wariantach:

- jako system mobilny lub stacjonarny,

- z jedną stacją główną i dwoma lub więcej stacjami podległymi,

- jako niezależne pary stacji.

Rysunki 1 i 2 przedstawiają konfiguracje stacji. Na obu rysunkach system jest zainstalowany na Zatoce Gdańskiej. System przedstawiony na rysunku 1 składa się ze stacji głównej zainstalowanej w Gdański oraz stacji podległych w Jastarni i Krynicy Morskiej.

Rysunek 2 przedstawia łańcuch składający się z dwóch par: Gdynia - Hel oraz Kuźnica - Górki Zachodnie. Takie wystawienie stacji tworzy optymalną konfigurację - minimalizującą HDOP (Horizontal Dilution of Precision), bardzo ważną z punktu widzenia bezpieczeństwa nawigacyjnego na Zatoce Gdańskiej. 


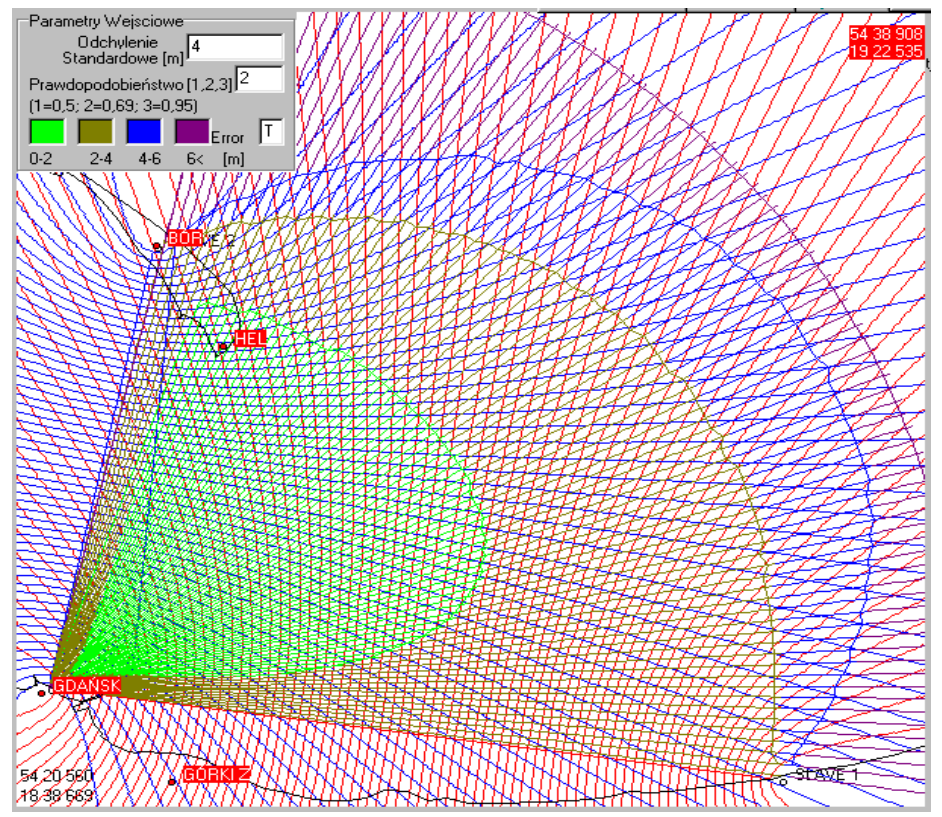

Rys. 1. Konfiguracja - jedna stacja główna i dwie podległe

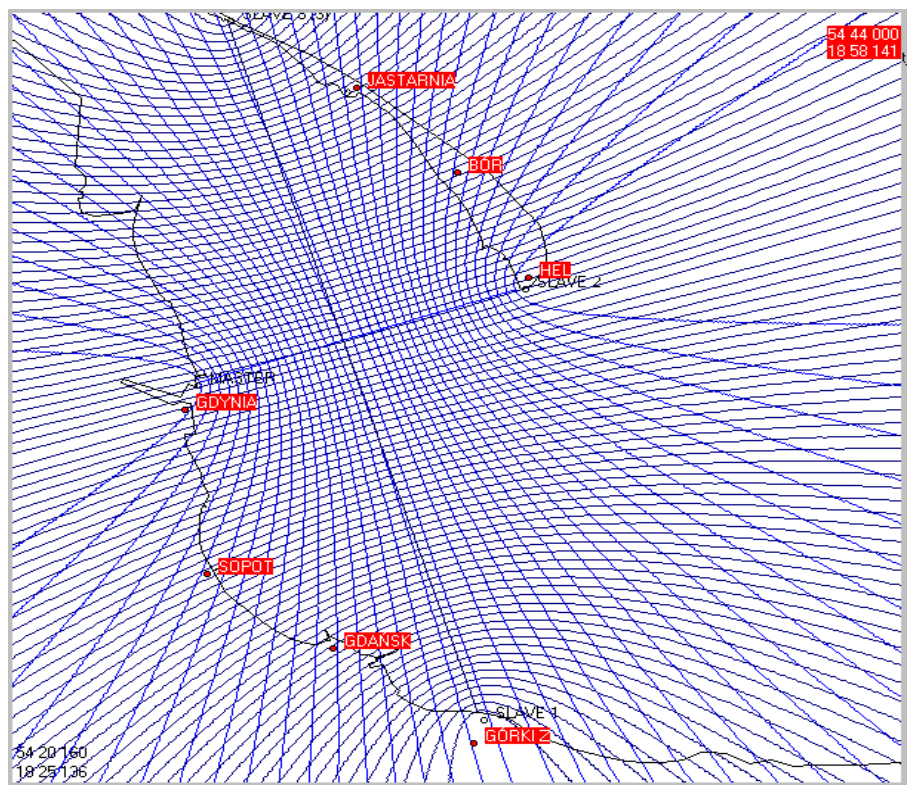

Rys. 2. Konfiguracja - dwie niezależne pary stacji 


\section{Parametry filtru}

Zgodnie z przyjętymi powyżej założeniami, w filtrze będą wykorzystywane pomiary następujących systemów i urządzeń nawigacyjnych [1]:

- satelitarnego systemu nawigacyjnego (D)GPS - współrzędne geograficzne pozycji $\left(\varphi_{D G P S}, \lambda_{D G P S}\right)$,

- naziemnego hiperbolicznego systemu radionawigacyjnego bliskiego zasięgu JEMIOLUSZKA - współrzędne geograficzne pozycji $\left(\varphi_{J E M}, \lambda_{J E M}\right)$,

- log dopplerowski - składowe prędkości względem dna $\left(V_{x}, V_{y}\right)$,

- żyrokompas - kurs $(C)$,

- przetwornik inercjalny - składowe przyspieszenia względem dna $\left(a_{x}, a_{y}\right)$.

Przy czym mierzone składowe prędkości i przyspieszenia są przeliczane na rzuty wektora prędkości względem dna na południk i równoleżnik $\left(V_{N}, V_{E}\right)$, rzuty wektora przyspieszenia względem dna na południk i równoleżnik $\left(a_{N}\right.$, $a_{E}$ ) oraz rzuty pochodnej przyspieszenia (jerk) względem dna na południk i równoleżnik $\left(j_{N}, j_{E}\right)$. Pochodne przyspieszenia przyjmujemy jako biały szum.

W przypadku tym wektor stanu będzie posiadał następujące elementy:

$$
\mathbf{x}=\left[\varphi, \lambda, V_{N}, V_{E}, a_{N}, a_{E}, j_{N}, j_{E}\right]^{\mathrm{T}}
$$

Zaś macierz przejścia A będzie określona następująco:

$$
\mathbf{A}_{i+1, i}=\left[\begin{array}{cccccccc}
1 & 0 & \Delta t_{i} & 0 & \Delta t_{i}{ }^{2} / 2 & 0 & \Delta t_{i}{ }^{3} / 6 & 0 \\
0 & 1 & 0 & \Delta t_{i} & 0 & \Delta t_{i}^{2} / 2 & 0 & \Delta t_{i}^{3} / 6 \\
0 & 0 & 1 & 0 & \Delta t_{i} & 0 & \Delta t_{i}{ }^{2} / 2 & 0 \\
0 & 0 & 0 & 1 & 0 & \Delta t_{i} & 0 & \Delta t_{i}^{2} / 2 \\
0 & 0 & 0 & 0 & 1 & 0 & \Delta t_{i} & 0 \\
0 & 0 & 0 & 0 & 0 & 1 & 0 & \Delta t_{i} \\
0 & 0 & 0 & 0 & 0 & 0 & 1 & 0 \\
0 & 0 & 0 & 0 & 0 & 0 & 0 & 1
\end{array}\right]
$$

gdzie: $\Delta t_{i}=t_{i+1}-t_{i}$ 
Teraz ewolucja stanu jest określona przez pochodne wyższych rzędów poszczególnych parametrów nawigacyjnych. Macierz kowariancji zakłóceń stanu ma postać dostosowaną do elementów wektora stanu. Tak więc będziemy mieli:

$$
\mathbf{Q}_{i}=\left[\begin{array}{cccccccc}
\sigma_{\varphi}^{2}+\Delta t_{i}{ }^{2} \sigma_{V_{\varphi}}^{2} & \Delta t_{i}{ }^{2} \sigma_{V_{\varphi} V_{\lambda}} & 0 & 0 & 0 & 0 & 0 & 0 \\
\Delta t_{i}{ }^{2} \sigma_{V_{\varphi} V_{\lambda}} & \sigma_{\lambda}^{2}+\Delta t_{i}{ }^{2} \sigma_{V_{\lambda}}^{2} & 0 & 0 & 0 & 0 & 0 & 0 \\
0 & 0 & \sigma_{V_{N}}^{2} & \sigma_{V_{N} V_{E}} & 0 & 0 & 0 & 0 \\
0 & 0 & \sigma_{V_{N} V_{E}} & \sigma_{V_{N}}^{2} & 0 & 0 & 0 & 0 \\
0 & 0 & 0 & 0 & \sigma_{a_{N}}^{2} & \sigma_{a_{N} a_{E}} & 0 & 0 \\
0 & 0 & 0 & 0 & \sigma_{a_{N} a_{E}} & \sigma_{a_{E}}^{2} & 0 & 0 \\
0 & 0 & 0 & 0 & 0 & 0 & \sigma_{j_{N}}^{2} & 0 \\
0 & 0 & 0 & 0 & 0 & 0 & 0 & \sigma_{j_{E}}^{2}
\end{array}\right]
$$

W tym modelu za wielkości mierzone przyjmijmy: współrzędne pozycji systemu DGPS $\left(\varphi_{D G P S}, \lambda_{D G P S}\right)$, współrzędne pozycji systemu JEMIOLUSZKA $\left(\varphi_{J E M}, \lambda_{J E M}\right)$, składowe prędkości względem południka i równoleżnika $\mathrm{z}$ nawigacji zliczeniowej $\left(V_{N}, V_{E}\right)$ oraz składowe przyspieszenia względem południka i równoleżnika $\mathrm{z}$ przetwornika inercjalnego $\left(a_{N}, a_{E}\right)$. Przy tych założeniach wektor pomiarów będzie wyglądał następująco:

$\mathbf{z}=\left[\varphi_{D G P S}, \lambda_{D G P S}, \varphi_{J E M}, \lambda_{J E M}, V_{N}, V_{E}, a_{N}, a_{E}\right]^{\mathrm{T}}$

Macierz pomiarów jest macierzą Jacobiego

$$
\mathbf{C}=\left[\begin{array}{cccccccc}
1 & 0 & 0 & 0 & 0 & 0 & 0 & 0 \\
0 & 1 & 0 & 0 & 0 & 0 & 0 & 0 \\
\frac{\partial \varphi_{J E M}}{\partial \varphi} & 0 & 0 & 0 & 0 & 0 & 0 & 0 \\
0 & \frac{\partial \lambda_{J E M}}{\partial \lambda} & 0 & 0 & 0 & 0 & 0 & 0 \\
0 & 0 & 1 & 0 & 0 & 0 & 0 & 0 \\
0 & 0 & 0 & 1 & 0 & 0 & 0 & 0 \\
0 & 0 & 0 & 0 & 1 & 0 & 0 & 0 \\
0 & 0 & 0 & 0 & 0 & 1 & 0 & 0
\end{array}\right]
$$


Zaś macierz kowariancji zakłóceń pomiarów (wektora pomiarów) w tym przypadku będzie wyglądała następująco:

$$
\mathbf{R}=\left[\begin{array}{cccccccc}
\sigma_{\varphi_{D G P S}}^{2} & \sigma_{\varphi \lambda_{D G P S}} & 0 & 0 & 0 & 0 & 0 & 0 \\
\sigma_{\varphi \lambda_{D G P S}} & \sigma_{\lambda_{D G P S}}^{2} & 0 & 0 & 0 & 0 & 0 & 0 \\
0 & 0 & \sigma_{\varphi_{J E M}}^{2} & \sigma_{\varphi \lambda_{J_{E M}}} & 0 & 0 & 0 & 0 \\
0 & 0 & \sigma_{\varphi \lambda_{J E M}} & \sigma_{\lambda_{J E M}}^{2} & 0 & 0 & 0 & 0 \\
0 & 0 & 0 & 0 & \sigma_{V_{N}}^{2} & \sigma_{V_{N} V_{E}} & 0 & 0 \\
0 & 0 & 0 & 0 & \sigma_{V_{N} V_{E}} & \sigma_{V_{E}}^{2} & 0 & 0 \\
0 & 0 & 0 & 0 & 0 & 0 & \sigma_{a_{N}}^{2} & \sigma_{a_{N} a_{E}} \\
0 & 0 & 0 & 0 & 0 & 0 & \sigma_{a_{N} a_{E}} & \sigma_{a_{E}}^{2}
\end{array}\right]
$$

\section{Dokładność pomiarów parametrów nawigacyjnych}

Jako elementy macierzy kowariancji występują wariancje i kowariancje poszczególnych pomiarów nawigacyjnych. Charakterystyki te zostały określone podczas prób statycznych (laboratoryjnych) i morskich. Rysunek 3 ukazuje przykład zmienności współrzędnych geograficznych określanych za pomocą DGPS.

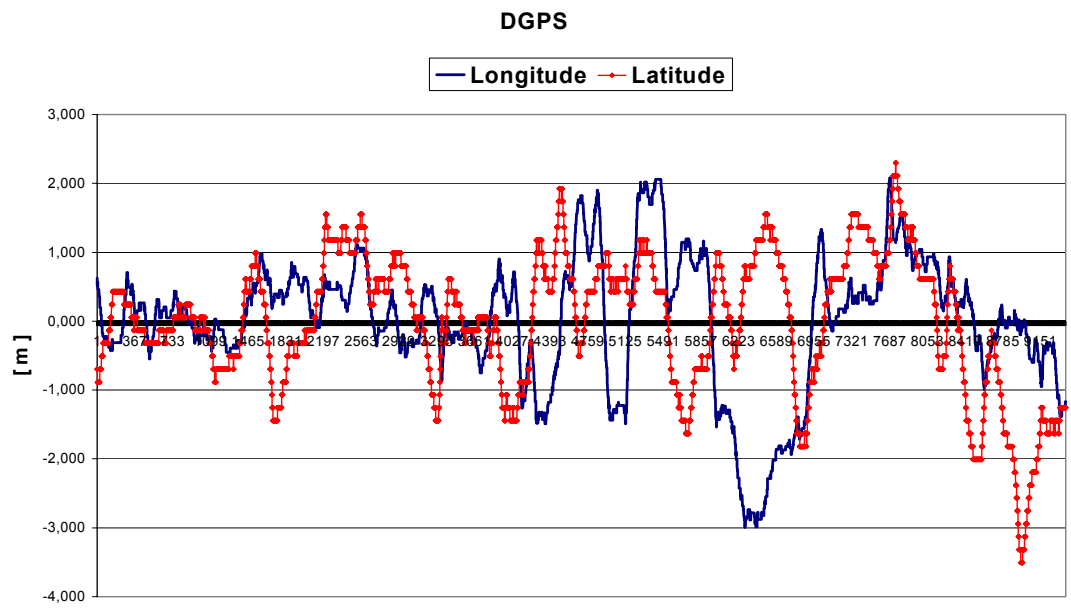

Rys. 3. Zmienność współrzędnych geograficznych DGPS

To samo $\mathrm{w}$ odniesieniu do systemu JEMIOLUSZKA pokazane jest na następnym rysunku (rys. 4). 


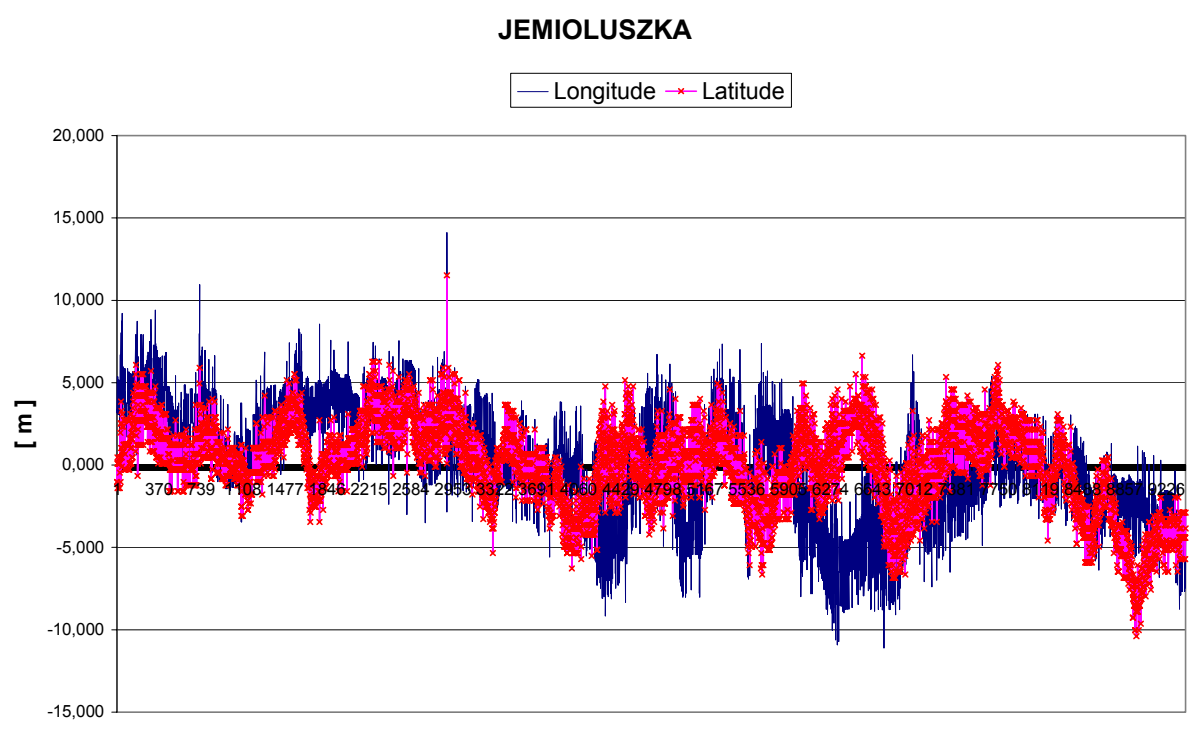

Rys. 4. Zmienność współrzędnych geograficznych systemu JEMIOLUSZKA

W efekcie przyjęto następujące wartości wariancji i kowariancji poszczególnych pomiarów:

- systemu DGPS $-\sigma_{\varphi_{D G P S}}^{2}=4,0\left[\mathrm{~m}^{2}\right], \sigma_{\lambda_{D G P S}}^{2}=2,25\left[\mathrm{~m}^{2}\right]$, współrzędne są nieskorelowane;

- składowych prędkości $-\sigma_{V}=0,1 \mathrm{~m} / \mathrm{s}$;

- $\quad$ składowych przyspieszeń $-\sigma_{a}=0,01 \mathrm{~m} / \mathrm{s}^{2}$;

- systemu JEMIOLUSZKA [2], [3]:

a) wariancja szerokości geograficznej

$$
\sigma_{\varphi_{J E M}}^{2}=\frac{1}{4} \sigma_{\Delta D}^{2} \csc ^{2} \theta\left(\cos ^{2} A_{12} \csc ^{2} \frac{\omega_{23}}{2}+\cos ^{2} A_{23} \csc ^{2} \frac{\omega_{12}}{2}\right)
$$

b) wariancja długości geograficznej

$$
\sigma_{\lambda_{J E M}}^{2}=\frac{1}{4} \sigma_{\Delta D}^{2} \csc ^{2} \theta\left(\sin ^{2} A_{12} \csc ^{2} \frac{\omega_{23}}{2}+\sin ^{2} A_{23} \csc ^{2} \frac{\omega_{12}}{2}\right),
$$

d) kowariancja pomiędzy współrzędnymi: 


$$
\sigma_{\varphi \lambda_{J E M}}=\frac{1}{8} \sigma_{\Delta D}^{2} \csc ^{2} \theta\left(\sin \left(A_{1}+A_{2}\right) \csc ^{2} \frac{\omega_{23}}{2}+\sin \left(A_{2}+A_{3}\right) \csc ^{2} \frac{\omega_{12}}{2}\right) ;
$$

gdzie:

$A_{i j} \quad$ - azymut pomiędzy $i$-tą oraz $j$-tą stacją,

$\omega_{i j}$ - kąt bazowy pomiędzy $i$-tą oraz $j$-tą stacją

$\sigma_{\Delta \mathrm{D}}-$ błąd pomiaru różnicy odległości,

$$
\theta=A_{i j}-A_{j k} .
$$

\section{Wnioski}

Przeprowadzono badania symulacyjne opracowanego algorytmu estymacji parametrów nawigacyjnych, a następnie próby morskie opracowanego systemu. Badania morskie potwierdziły poprawność pracy filtru i jego przydatność w algorytmie INS specjalistycznych okrętów. Rysunek 5 pokazuje porównanie pozycji otrzymywanych z zintegrowanego systemu nawigacyjnego (INS) z pomiarami systemów pozycyjnych - DGPS i JEMIOLUSZKA.

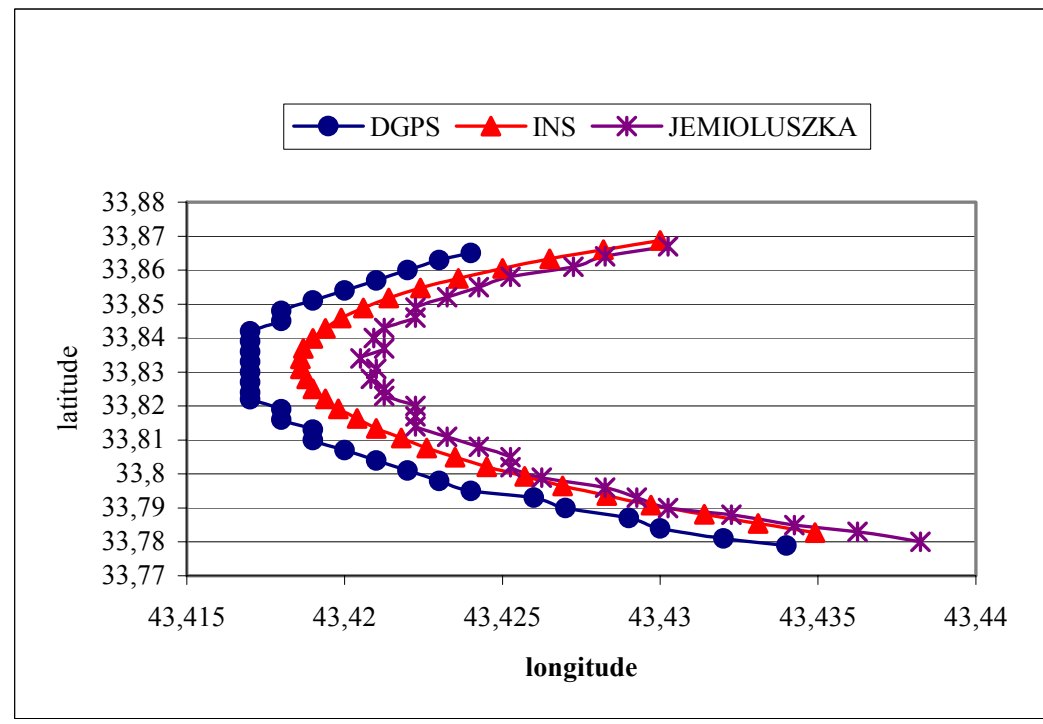

Rys. 5. Porównanie pozycji DGPS, JEMIOLUSZKA i INS na cyrkulacji

Przedstawiony powyżej model procesu nawigacyjnego nie wyczerpuje wszystkich możliwych rozwiązań. Należy wyraźnie podkreślić, że postać 
modeli decyduje w dużym stopniu o sukcesie lub porażce opracowywanego filtru. Dotyczy to przede wszystkim poprawnego, adekwatnego do rzeczywistości określenia elementów poszczególnych macierzy - przejścia $\mathbf{A}$, pomiarów $\mathbf{C}$ oraz macierzy kowariancji $\mathbf{Q}$ i $\mathbf{R}$. W przypadku macierzy kowariancji najistotniejszy jest stosunek odpowiednich elementów względem siebie. Przyjęte zbyt duże błędy zakłóceń stanu powodują, że filtr staje się ,sztywny” i za mocno nadąża za pomiarami [1], [4]. Efektem tego jest to, że nie są odfiltrowywane błędy pomiarów. Gdy z kolei przyjmiemy zbyt małe wartości zakłóceń stanu, wtedy filtr zacznie odrzucać pomiary zbyt mocno różniące się od ich prognoz. Szczególnie ważne jest to $\mathrm{w}$ rzeczywistych sytuacjach pomiarowych, gdy występuje niezgodność pomiędzy pomiarami z różnych urządzeń i systemów nawigacyjnych oraz przy niskiej wiarygodności mierników oraz wyników pomiarów. Nie można również zapominać o problemie synchronizacji skal czasu poszczególnych mierników, długości cykli pomiarowych oraz przedziałach dyskretyzacji.

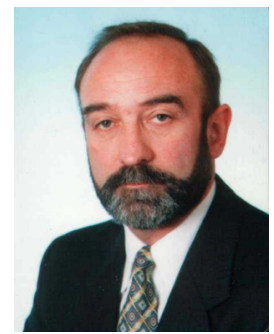

Andrzej Banachowicz, comdr (Ret.), Ph.D., DSc. and professor of Gdynia Maritime University. Graduated from Naval University of Gdynia in 1976 and from University of Gdansk (in mathematics) in 1984. Since 1976 to 1995 he worked in Naval University of Gdynia. Since 1995 he is a professor of Gdynia Maritime University. Fields of educational and research activity: maritime navigation, navigational mathematics and automation and computerization of navigational processes. Author and co-author more than 180 publications. 